\title{
Radiation From A Line Source Shielded By A Confocal Elliptic Layer Of DNG Metamaterial
}

\author{
Oguzhan Akgol, Vito G. Daniele, Danilo Erricolo, Senior Member, IEEE, Piergiorgio L. E. Uslenghi, Life Fellow, \\ IEEE.
}

\begin{abstract}
The two-dimensional problem of electromagnetic radiation from a line source located inside an elliptic-cylinder confocal sheath of double-negative metamaterial is solved exactly by separation of variables. Numerical results are presented and discussed for the radiation pattern and the field inside the sheath, as functions of frequency, location of the line source, and sheath thickness and eccentricity.
\end{abstract}

Index Terms-Electromagnetic radiation, exact solution, metamaterial, shielding.

\section{INTRODUCTION}

A Confocal elliptic cylindrical layer made of doublenegative (DNG) metamaterial is considered. The space inside and outside the layer is filled with a lossless homogeneous double-positive (DPS) medium characterized by a real positive electric permittivity $\epsilon_{1}$ and a real positive magnetic permeability $\mu_{1}$, whereas the DNG sheath is characterized by a real negative permittivity $-\epsilon_{2}$ and a real negative permeability $-\mu_{2}$. The isorefractive condition $\epsilon_{1} \mu_{1}=\epsilon_{2} \mu_{2}$ is assumed. Consequently, the wavenumber is

$$
k_{\ell}=-(-1)^{\ell} \omega \sqrt{\epsilon_{\ell} \mu_{\ell}},
$$

where $\omega$ is the angular frequency, and $\ell=1$ in the DPS regions of space and $\ell=2$ inside the DNG sheath, meaning that the real positive wavenumber in the DPS regions has the same magnitude but opposite sign to the real negative wavenumber in the DNG layer. The real positive intrinsic impedances are

$$
Z_{\ell}=\sqrt{\frac{\mu_{\ell}}{\epsilon_{\ell}}}
$$

The primary field is an electric or magnetic line source parallel to the generators of the cylindrical sheath and located anywhere inside the DPS medium encased by the sheath. The analysis is conducted in phasor domain with the timedependence factor $\exp (+j \omega t)$ omitted throughout. In all regions, the field components are expressed as infinite series of Mathieu functions whose modal expansion coefficients are found analytically by imposing the boundary conditions.

This novel analytical solution is evaluated numerically for different values of the parameters involved: dimensions and thickness of the sheath in terms of wavelength, eccentricity of the elliptical cross-section of the sheath, ratio of the intrinsic impedances of the DPS and DNG media, location of the line source. Particular attention is devoted to the radiated far field and to the field inside the DNG layer.

O. Akgol, D. Erricolo and P.L.E. Uslenghi are with the Department of ECE, University of Illinois at Chicago, IL 60607, USA. (e-mails: oakgol2@uic.edu, erricolo@ece.uic.edu, uslenghi@uic.edu).V.G. Daniele is with Dipartimento di Elettronica, Politecnico di Torino, Italy. (e-mail: vito.daniele@polito.it).
A simplified solution is obtained when the intrinsic impedances of the DPS and DNG media are equal and the source is located in the plane containing the focal lines of the elliptical sheath. In particular, a comparison with a geometrical-optics solution is performed when the source coincides with a focal line; this simplified solution was presented at a conference [1]. Previous works have examined the case of an elliptic cylinder completely filled with DNG metamaterial and excited by a line source located at a focal line, both from an optical [2] and an electromagnetic [3] viewpoint.

\section{GEOMETRY OF THE PROBLEM}

A cross-section of the structure is shown in Fig. 1. The generators of the elliptic-cylindrical sheath are parallel to the $z$-axis, and the elliptic cylinder coordinates $(u, v, z)$ are related to the rectangular coordinates $(x, y, z)$ by $x=$ $d / 2 \cosh u \cos v, y=d / 2 \sinh u \sin v, z=z$, where $0 \leq u<$ $\infty, 0 \leq v \leq 2 \pi,-\infty<z<\infty$ and $d$ is the interfocal distance between the focal lines $F_{1}$ and $F_{2}$. The dimensionless parameters

$$
c=k_{1} d / 2=\pi d / \lambda, \quad \zeta=Z_{1} / Z_{2},
$$

where $\lambda$ is the wavelength, are introduced. The inner and outer surfaces of the DNG sheath correspond to $u=u_{1}$ and $u=u_{2}$, respectively. The position of the line source is indicated by $S$ in Fig. 1. Because of symmetries, we may restrict its location to the first quadrant, corresponding to coordinates $u_{0}$ and $v_{0}$ such that $0 \leq u_{0} \leq u_{1}, 0 \leq v_{0} \leq \pi / 2$.

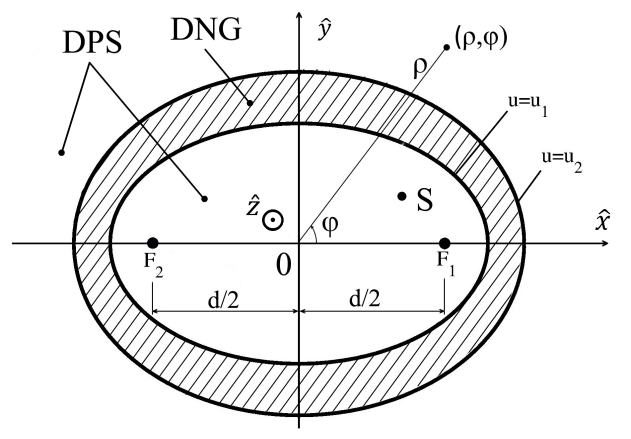

Fig. 1. Cross-section of the structure 


\section{Analytical SOlution}

Consider an isotropic electric line source with electric field

$$
\boldsymbol{E}^{i}=\hat{z} E_{1 z}^{i}=\hat{z} H_{0}^{(2)}(k R)
$$

where $H_{0}^{(2)}$ is the Hankel function of the second kind and $R$ is the distance of the observation point from the line source. This field may be expanded in an infinite series of products of radial and angular Mathieu functions [4]:

$$
\begin{aligned}
& E_{1 z}^{i}=4 \sum_{m=0}^{\infty} {\left[\frac{\operatorname{Re}_{m}^{(1)}\left(c, u_{<}\right) \operatorname{Re}_{m}^{(4)}\left(c, u_{>}\right) \operatorname{Se}_{m}\left(c, v_{0}\right) \operatorname{Se}_{m}(c, v)}{N_{m}^{(e)}}\right.} \\
&+\left.\frac{\operatorname{Ro}_{m}^{(1)}\left(c, u_{<}\right) \operatorname{Ro}_{m}^{(4)}\left(c, u_{>}\right) \operatorname{So}_{m}\left(c, v_{0}\right) \operatorname{So}_{m}(c, v)}{N_{m}^{(o)}}\right] \\
&\left.\left(u_{<}, u_{>}\right) \leq u_{1}\right)
\end{aligned}
$$

where $u i_{<}\left(u_{>}\right)$is the smaller (larger) between $u$ and $u_{0}$. The total electric field in the DPS region enclosed by the DNG sheath is

$$
E_{1 z}=E_{1 z}^{i}+E_{1 z}^{d}
$$

where

$$
\begin{aligned}
E_{1 z}^{d}=4 \sum_{m=0}^{\infty} & {\left[\frac{a_{m}^{(e)}}{N_{m}^{(e)}} \operatorname{Re}_{m}^{(1)}\left(c, u_{0}\right) \operatorname{Re}_{m}^{(1)}(c, u) \operatorname{Se}_{m}\left(c, v_{0}\right) \operatorname{Se}_{m}(c, v)+\right.} \\
& \left.\frac{a_{m}^{(o)}}{N_{m}^{(o)}} \operatorname{Ro}_{m}^{(1)}\left(c, u_{0}\right) \operatorname{Ro}_{m}^{(1)}(c, u) \operatorname{So}_{m}\left(c, v_{0}\right) \operatorname{So}_{m}(c, v)\right] \\
& \left(u \leq u_{1}\right)
\end{aligned}
$$

The electric field radiated into the infinite DPS space surrounding the structure is

$$
\begin{aligned}
& E_{1 z}^{s}=4 \sum_{m=0}^{\infty} \\
& {\left[\frac{c_{m}^{(e)}}{N_{m}^{(e)}} \operatorname{Re}_{m}^{(1)}\left(c, u_{0}\right) \operatorname{Re}_{m}^{(4)}(c, u) \operatorname{Se}_{m}\left(c, v_{0}\right) \operatorname{Se}_{m}(c, v)+\right.} \\
& \left.\frac{c_{m}^{(o)}}{N_{m}^{(o)}} \operatorname{Ro}_{m}^{(1)}\left(c, u_{0}\right) \operatorname{Ro}_{m}^{(4)}(c, u) \operatorname{So}_{m}\left(c, v_{0}\right) \operatorname{So}_{m}(c, v)\right] \\
& \left(u \geq u_{2}\right)
\end{aligned}
$$

The electric field inside the DNG layer is

$$
\begin{aligned}
& E_{2 z}=4 \sum_{m=0}^{\infty}\left[\frac { \operatorname { R e } _ { m } ^ { ( 1 ) } ( c , u _ { 0 } ) } { N _ { m } ^ { ( e ) } } \left[b_{m}^{(e)} \operatorname{Re}_{m}^{(1)}(-c, u)+\right.\right. \\
& \left.d_{m}^{(e)} \operatorname{Re}_{m}^{(4)}(-c, u)\right] \operatorname{Se}_{m}\left(c, v_{0}\right) \operatorname{Se}_{m}(c, v)+\frac{\operatorname{Ro}_{m}^{(1)}\left(c, u_{0}\right)}{N_{m}^{(o)}} \\
& \left.\left[b_{m}^{(o)} \operatorname{Ro}_{m}^{(1)}(-c, u)+d_{m}^{(o)} \operatorname{Ro}_{m}^{(4)}(-c, u)\right] \operatorname{Se}_{m}\left(c, v_{0}\right) \operatorname{Se}_{m}(c, v)\right] \\
& \left(u_{1} \leq u \leq u_{2}\right)
\end{aligned}
$$

where the fact that the angular functions $S e_{m}$ and $S o_{m}$ are even functions of the parameter $c$ has been taken into account.

The modal expansion coefficients $a_{m}^{(e, o)}, b_{m}^{(e, o)}, d_{m}^{(e, o)}$ and $c_{m}^{(e, o)}$ are determined by imposing the boundary conditions, i.e. the continuity of the tangential field components $E_{z}$ and
$H_{v}$ accross the interfaces $u=u_{1}$ and $u=u_{2}$. The magnetic field component $H_{v}$ is related to $E_{z}$ by

$$
H_{l v}=\frac{(-1)^{l} j}{c Z_{l}} \frac{1}{\sqrt{\cosh ^{2} u-\cos ^{2} v}} E_{l z}^{\prime},
$$

where $l=1(2)$ for DPS (DNG) media, and here and in the following the prime means derivative with respect to $u$. It is found that

$$
\begin{aligned}
& a_{m}^{(e)}=\frac{1}{\Delta^{(e)}}\left[A_{m}^{(e)}\left(u_{1}\right) B_{m}^{(e)}\left(u_{2}\right)-A_{m}^{(e)}\left(u_{2}\right) B_{m}^{(e)}\left(u_{1}\right)\right], \\
& b_{m}^{(e)}=\frac{-j B_{m}^{(e)}\left(u_{2}\right)}{\Delta^{(e)}}, c_{m}^{(e)}=\frac{-\zeta}{\Delta^{(e)}}, d_{m}^{(e)}=\frac{j A_{m}^{(e)}\left(u_{2}\right)}{\Delta^{(e)}}
\end{aligned}
$$

where

$$
\begin{aligned}
& A_{m}^{(e)}(u)= \\
& \operatorname{Re}_{m}^{(1)}(-c, u) \operatorname{Re}_{m}^{(4)^{\prime}}(c, u)+\zeta \operatorname{Re}_{m}^{(1)^{\prime}}(-c, u) \operatorname{Re}_{m}^{(4)}(c, u), \quad(13) \\
& B_{m}^{(e)}(u)= \\
& \operatorname{Re}_{m}^{(1)}(-c, u) \operatorname{Re}_{m}^{(4)^{\prime}}(c, u)+\zeta \operatorname{Re}_{m}^{(4)^{\prime}}(-c, u) \operatorname{Re}_{m}^{(4)}(c, u), \quad(14) \\
& \Delta^{(e)}=A_{m}^{(e)}\left(u_{2}\right)\left[\operatorname{Re}_{m}^{(1)^{\prime}}\left(c, u_{1}\right) \operatorname{Re}_{m}^{(4)}\left(-c, u_{1}\right)+\zeta \operatorname{Re}_{m}^{(1)}\left(c, u_{1}\right)\right. \\
& \left.\times \operatorname{Re}_{m}^{(4)^{\prime}}\left(-c, u_{1}\right)\right]-B_{m}^{(e)}\left(u_{2}\right)\left[\operatorname{Re}_{m}^{(1)^{\prime}}\left(c, u_{1}\right) \operatorname{Re}_{m}^{(1)}\left(-c, u_{1}\right)+\right. \\
& \left.\zeta \operatorname{Re}_{m}^{(1)}\left(c, u_{1}\right) \operatorname{Re}_{m}^{(1)^{\prime}}\left(-c, u_{1}\right)\right] .
\end{aligned}
$$

The coefficients $a_{m}^{(o)}, b_{m}^{(o)}, d_{m}^{(o)}$ and $c_{m}^{(o)}$ are obtained by replacing $\mathrm{Re}_{m}^{(1,4)}$ and their derivatives with $\mathrm{Ro}_{m}^{(1,4)}$ and their derivatives everywhere in the expressions for $a_{m}^{(o)}, b_{m}^{(o)}, d_{m}^{(o)}$ and $c_{m}^{(o)}$ respectively.

In the absence of a DNG sheath, it is easily verified that

$$
\left.a_{m}^{(e, o)}\right|_{\xi_{1}=\xi_{2}}=0,\left.\quad c_{m}^{(e, o)}\right|_{\xi_{1}=\xi_{2}}=1,
$$

as should be expected. With reference to circular cylindrical coordinates $(\rho, \phi, z)$ (see Fig.1), the radiated far field is

$$
\left.E_{1 z}^{s}\right|_{k \rho \rightarrow \infty} \approx P^{(e)}(\varphi) \frac{e^{-j k \rho+j \frac{\pi}{4}}}{\sqrt{k \rho}},
$$

where the far-field coefficient is

$$
\begin{aligned}
P^{(e)}(\varphi)= & 4 \sum_{m=0}^{\infty} j^{m}\left[\frac{c_{m}^{(e)}}{N_{m}^{(e)}} \operatorname{Re}_{m}^{(1)}\left(c, u_{0}\right) \operatorname{Se}_{m}\left(c, v_{0}\right) \operatorname{Se}_{m}(c, \varphi)\right. \\
& \left.+\frac{c_{m}^{(o)}}{N_{m}^{(o)}} \operatorname{Ro}_{m}^{(1)}\left(c, u_{0}\right) \operatorname{So}_{m}\left(c, v_{0}\right) \operatorname{So}_{m}(c, \varphi)\right]
\end{aligned}
$$

In the absence of the DNG sheath, the far-field coefficient is that of the line source (6), and is equal to one. Hence, the farfield power pattern normalized to the power pattern without the DNG sheath is

$$
P(\varphi)=\left|P^{(e)}(\varphi)\right|^{2} .
$$

Simplifications in the formulas of this section occur when the line source is located either in the $x=0$ plane or in the $y=0$ plane of Fig.1. In particular, if the source coincides with the 
focal line $F_{1}$ of Fig.1, so that $u_{0}=v_{0}=0$, the far-field coefficient (18) simpifies to:

$$
\left.P^{(e)}(\varphi)\right|_{u_{0}=v_{0}=0}=4 \sum_{m=0}^{\infty} \frac{j^{m}}{N_{m}^{(e)}} c_{m}^{(e)} \operatorname{Re}_{m}^{(1)}(c, 1) \operatorname{Se}_{m}(c, \varphi) .
$$

The solution for an isotropic magnetic line source is obtained by duality from the solution for an electric line source.

\section{A Geometrical Optics Solution}

Consider the particular case of an isotropic line source located at the focal line $F_{1}$. With reference to the cross-section of Fig. 2, the optical ray $F_{1} A$ is totally transmitted across the interface $u=u_{1}$ in the direction $A B$, as though it were produced by an anisotropic virtual line source located at the other focal line $F_{2}$. Along $\mathrm{AB}$, the normalized power emitted per unit angle is [2]

$$
p(\beta)=\frac{1-\chi_{1}^{2}}{1+\chi_{1}^{2}-2 \chi_{1} \cos \beta},
$$

where $\beta$ is the angle that the direction $F_{2} A B$ of propagation forms with the positive $\mathrm{x}$-axis. The eccentricity of the crosssectional ellipse $u=u_{\ell}$ is

$$
\chi_{\ell}=\frac{d}{2 a_{\ell}},(\ell=1,2)
$$

where $a_{\ell}$ is the semi-major axis of the ellipse.

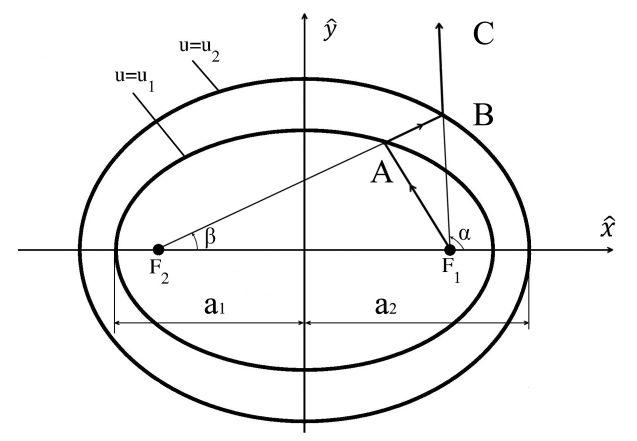

Fig. 2. Geometry of the problem for the geometrical optics solution.

The optical ray $A B$ is totaly transmitted across the interface $u=u_{2}$ in the direction $F_{1} B C$. If the virtual line source at $F_{2}$ were isotropic, the normalized power emitted per unit angle in the direction $B C$ would be

$$
p_{\text {iso }}=\frac{1-\chi_{2}^{2}}{1+\chi_{2}^{2}+2 \chi_{2} \cos \alpha},
$$

where $\alpha$ is the angle that the direction $F_{1} B C$ forms with the positive $\mathrm{x}$-axis. Therefore, the optical power emitted per unit angle along $B C$, normalized to the power emitted isotropically per unit angle by the line source at $F_{1}$, is

$$
\begin{aligned}
p(\alpha)= & p_{\text {iso }}(\alpha) p(\beta)= \\
& \frac{\left(1-\chi_{1}^{2}\right)\left(1-\chi_{2}^{2}\right)}{\left(1+\chi_{1}^{2}-2 \chi_{1} \cos \beta\right)\left(1+\chi_{2}^{2}+2 \chi_{2} \cos \alpha\right)} .
\end{aligned}
$$

To complete the optical solution, it remains to express $\beta$ as a function of $\alpha$. After some geometrical manipulations, it is found that

$$
\cos \beta=\frac{\left(1+\chi_{2}^{2}\right) \cos \alpha+2 \chi_{2}}{\left.1+\chi_{2}^{2}+2 \chi_{2} \cos \alpha\right)} .
$$

\section{Numerical Results}

We first examine comparisons between the radiated power pattern given by eq. (19) and the geometrical optical solution of eq. (24). The results are parameterized according to the ratio $r=2 a_{1} / \lambda$. A smaller value of the ratio correspond to an electrically small cross-section, while a large value of the ratio is associated with an electrically large cross-section, up to infinity that corresponds to the geometrical optics case. Referring to Fig. 3, when $r=0.05$ the radiated power pattern is a slightly flattened circle; when $r=0.1$, the flattening becomes more prominent; and, when $r=4.3$ the power radiated pattern approaches the geometrical optics solution.

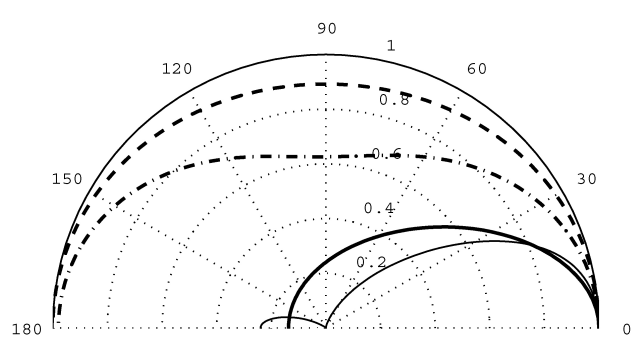

Fig. 3. Normalized radiated power patterns for a structure with $u_{1}=0.3$, $u_{2}=0.5, \zeta=10$. The dashed line represents $r=0.05$ (or $c=0.15$ ); the dash-dot line represents $r=0.1$ (or $c=0.3$ ); the thin solid line represents $r=4.3$ (or $c=12.92$ ); and the thick solid line represents the GO solution.

Next, we examine the effect of changing $\zeta$, while keeping constant all other parameters. Fig. 4 indicates quite significant changes of the normalized radiated power pattern of (19) when $\zeta=0.1,1,10$.

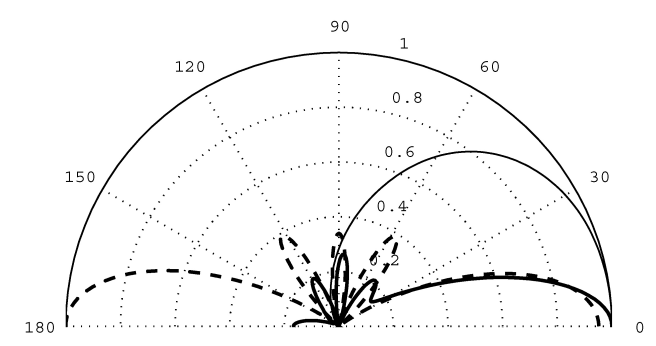

Fig. 4. Effect of the variation of the parameter $\zeta$ of eq. (3) for a structure with $u_{1}=0.5, u_{2}=0.8$ and a line source located at $u_{0}=0.25, v_{0}=0$, with $r=2$. Normalized radiated power patterns for $\zeta=0.1$, dashed line; $\zeta=1$, thin solid line; and $\zeta=10$ thick solid line 

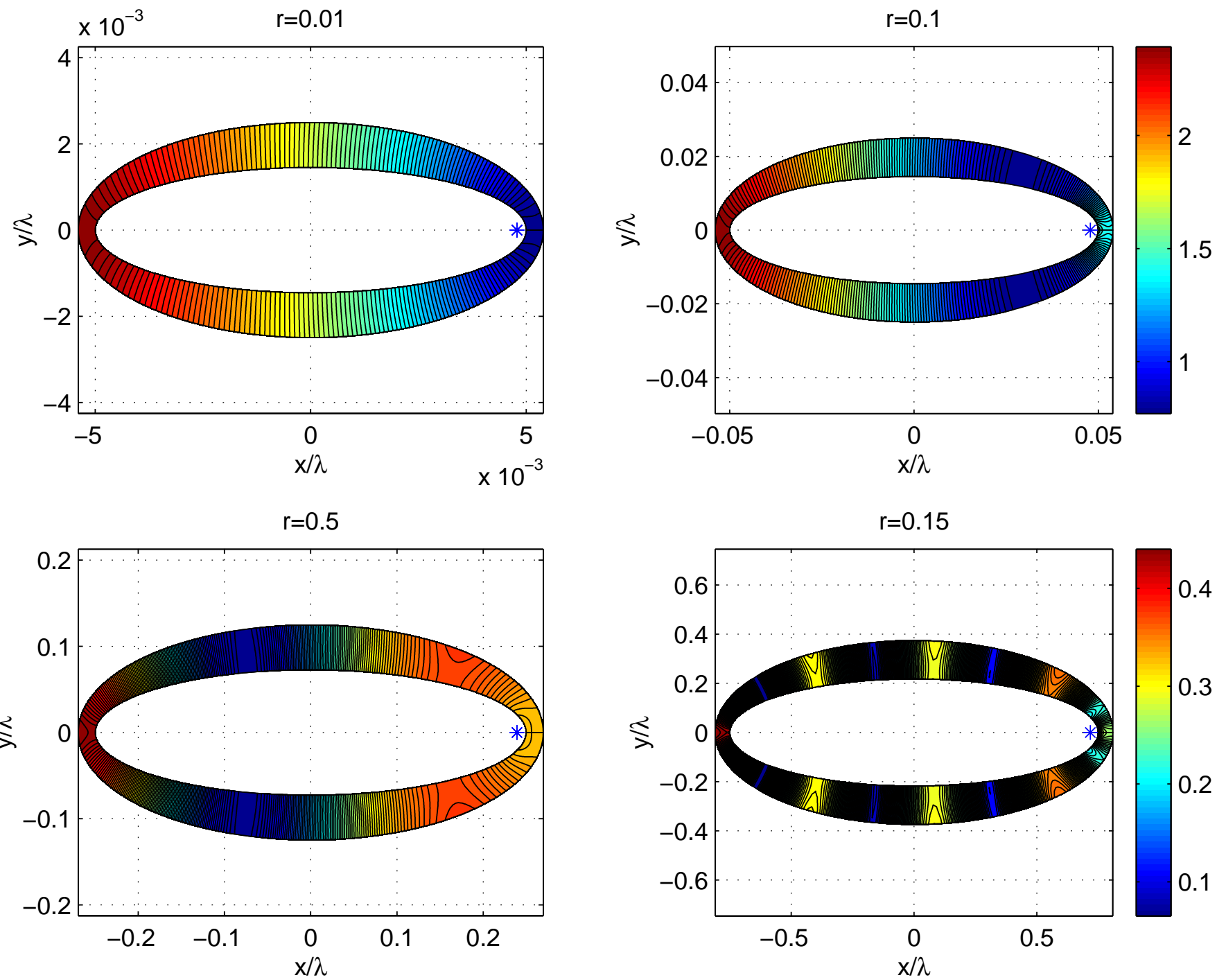

Fig. 5. Behavior of $\left|E_{z}\right|$ within the DNG layer with $u_{1}=0.3, u_{2}=0.5, \zeta=10$ for various values of the ratio $r$ and for a source located at $u_{0}=0, v_{0}=0$.

The last numerical example is the behavior of the magnitude of the the electric field within the DNG, which is represented in Fig. 5 for various values of the ratio $r$. For smaller values of $r$ there are fewer oscillations within the DNG layer, while the behavior becomes more complex for larger values of $r$.

The computations of the Mathieu functions were carried out with an extension of the software described in [5] and the acceleration technique described in [6].

\section{CONCLUSION}

The novel two-dimensional boundary value problem of radiation from a line source shielded by a confocal elliptic layer of DNG metamaterial has been solved exactly. Numerical data for the radiated far-field and the field inside the sheath have been exhibited.

\section{REFERENCES}

[1] P.L.E. Uslenghi, O. Akgol, V. G Daniele, and D. Erricolo, "Exact radiation by a line source located inside a confocal elliptic layer of DNG metamaterial," in XXX URSI General Assembly and Scientific Symposium, Istanbul, Turkey, Aug. 13-20, 2011.

[2] P.L.E. Uslenghi, "Optical behavior of elliptical lenses made of dng metamaterial," IEEE Antennas Wirel. Propag. Lett., vol. 9, pp. 566-567, 2010.

[3] O. Akgol, D. Erricolo, and P.L.E Uslenghi, "Exact imaging by an elliptic lens," IEEE Antennas Wirel. Propag. Lett., vol. 10, pp. 639-642, 2011.

[4] J. J. Bowman, T. B. A. Senior, and P. L. E. Uslenghi, Electromagnetic and Acoustic Scattering by Simple Shapes, Hemisphere Publishing Corporation, New York, 1987.

[5] D. Erricolo, "Algorithm 861: Fortran 90 subroutines for computing the expansion coefficients of Mathieu functions using Blanch's algorithm," ACM Transactions on Mathematical Software, vol. 32, no. 4, pp. 622634, Dec. 2006.

[6] D. Erricolo, "Acceleration of the convergence of series containing Mathieu functions using Shanks transformation," IEEE Antennas Wirel. Propag. Lett., vol. 2, pp. 58-61, 2003. 